\title{
Visibilidad de las revistas latinoamericanas de bibliotecología y ciencia de la información a través de Google Scholar
}

\author{
Sandra Miguel \\ Universidad Nacional de La Plata. Doctora en \\ documentación, Universidad de Granada. Licenciada en \\ bibliotecología y ciencia de la información. Profesora e \\ investigadora de la Universidad Nacional de La Plata. La \\ Plata, Argentina. Integrando del Grupo SCImago, unidad \\ asociada al CSIC, Buenos Aires, Argentina. \\ E-mail: sandra@fcnym.unlp.edu.ar
}

\section{Víctor Herrero_Solana}

Doctor en documentación. Universidad de Granada, Departamento de Biblioteconomía - CSIC, unidad asociada Grupo SCImago - Granada, España

E-mail: victorhs@ugr.es

\section{Resumen}

Las revistas académico-científicas son uno de los principales canales de comunicación y difusión de los resultados de investigación. Sin embargo no todas tienen el mismo prestigio y grado de influencia en la comunidad científica. De su calidad y visibilidad dependen, en gran medida, las posibilidades de que un trabajo sea conocido, leído y citado. Esta investigación se propone determinar la visibilidad en Google Scholar de un conjunto de revistas latinoamericanas de Bibliotecología y Ciencia de la Información, y clasificarlas según su grado de visibilidad. Utilizando la aplicación Publish or Perish para recuperar las citas de las revistas se calcularon indicadores basados en la citación. Se combinaron técnicas de análisis multivariado de datos para realizar agrupamientos de revistas según su grado de visibilidad, y se elaboraron representaciones gráficas que facilitan la interpretación de los resultados y la identificación de los grupos de revistas con alta, media y baja visibilidad.

\section{Palabras clave}

Visibilidad; Revistas; Bibliotecología y Ciencia de la Información; América Latina y el Caribe; Google Scholar.

\author{
Visibility of Latin American journals of \\ Library and Information Science from \\ Google Scholar
}

\begin{abstract}
The academic-scientific journals are one of the outstanding means of communication and diffusion of the investigation results. Nevertheless they don't have all the same prestige and degree of influence in the scientific community. The possibilities that a work is well-known, read and cited depend on its quality and visibility. The aim of this investigation is to determine the visibility in Google Scholar of a set of Latin-American Library and Information Science journals, and to classify them according to its degree of visibility. Using the Publish or Perish application to recover journal cites, indicators based on citation were estimated. Techniques of multivaried analysis of data were combined to make groups of journals according to their degree of visibility. Finally, graphical representations that facilitate the interpretation of the results and the identification of the groups of journals with high, mean and low visibility were elaborated.
\end{abstract}

\section{Keywords}

Visibility; Journals; Library and Information Science; Latin America and Caribbean; Google Scholar.

\section{INTRODUCCIÓN}

Está ampliamente aceptado que las revistas académico-científicas son uno de los principales canales de comunicación y difusión de los resultados de investigación. Sin embargo, no todas tienen el mismo prestigio y grado de influencia en la comunidad científica (Martin y Irvine, 1983). De su calidad y visibilidad dependen en gran medida las posibilidades de que un trabajo sea conocido, leído y citado. 
La calidad de las revistas está relacionada principalmente al cumplimiento de un conjunto de pautas editoriales, de presentación, de gestión y de contenidos que garanticen el rigor científico de los artículos publicados en ellas, y por tanto abonen a su prestigio. La visibilidad, en cambio, está dada por la recepción dispensada a los trabajos por el resto de la comunidad académica y científica, tras su publicación (Maltrás Barba, 2003).

Si bien se trata de dos aspectos conceptualmente diferentes, están íntimamente relacionados, a tal punto que algunos autores sostienen que la visibilidad, entendida en términos de la cantidad de citas recibidas por las revistas, es un indicador de su calidad científica que se suma a los anteriores (Herrero-Solana y Liberatore, 2008). Por otra parte, la visibilidad se potencia cuando las revistas son incluidas en bases de datos bibliográficas, y más aún cuando éstas tienen alcance internacional; en tanto que, la calidad constituye el filtro selectivo que establecen los editores y distribuidores de esas bases de datos para la inclusión de las revistas en dichas fuentes.

Las bases de datos del Institute for Scientific Information (ISI, ahora Thomson Scientific, de Philadelphia), han sido durante décadas las fuentes de datos más utilizadas para medir la visibilidad científica de las revistas, a partir del análisis de citación y del indicador factor de impacto. En la actualidad, se han empezado a utilizar también otras fuentes de datos complementarias como SCOPUS, de Elsevier, y Google Scholar (GS) de Google, a la vez que se han ido desarrollando algunos indicadores alternativos al factor de impacto ISI. Como ejemplos podemos citar el SJR (Scimago Journal Rank) desarrollado por el grupo SCImago, que refleja la visibilidad de las revistas contenidas en la base de datos SCOPUS (Scimago, 2007); el índice h, propuesto por Hirsch (2005), como una medida de la trayectoria científica de los investigadores, y varias otras medidas alternativas y complementarias del mismo que utilizaremos en este estudio y describiremos en el apartado Materiales y métodos.

\section{LAS REVISTAS LATINOAMERICANAS DE BIBLIOTECOLOGÍA Y CIENCIA DE LA INFORMACIÓN}

Si bien desde hace varios años, la literatura científica de Bibliotecología y Ciencia de la Información (ByCI) procedente de los países latinoamericanos ${ }^{1}$ revela tendencias de crecimiento en cuanto a su producción (Herrero-Solana y Ríos-Gómez, 2006), la investigación es aún una actividad de desarrollo incipiente. Asimismo, y en lo que a las revistas se refiere, se evidencian dificultades para su identificación, determinación de su naturaleza, valoración de su calidad e inclusión en los grandes servicios de indización y resúmenes (Gómez Fuentes, 1993; Ramírez Leyva, 1994); factores todos que dificultan enormemente sus posibilidades de visibilidad y reconocimiento internacional.

En los últimos años se han puesto en marcha numerosas iniciativas para elevar la visibilidad de las revistas procedentes de la región latinoamericana e iberoamericana. Proyectos como el Sistema Regional de Información en Línea para Revistas Científicas de América Latina, el Caribe, España y Portugal (LATINDEX), el Scientific Electronic Library Online (SCielo) y la Red de Revistas Científicas de América Latina y el Caribe (Red ALyC), son algunos ejemplos de los esfuerzos que hoy contribuyen al conocimiento y difusión de las revistas de la región en los distintos campos del conocimiento, incluyendo las de ByCI. También se han realizado diversos estudios con fines de identificar las revistas de la especialidad editadas en los países de América Latina y el Caribe, como los de García León (2001) y Urbizagástegui Alvarado (2006), entre otros.

El principal inconveniente a la hora de estudiar la producción científica procedente de la región, así como de determinar la visibilidad de las revistas se encuentra en las propias fuentes de datos. Hasta hace poco, no había revistas latinoamericanas de ByCI en el ISI. Lo único que podía verse en esta base de

${ }^{1}$ Utilizamos el término latinoamericano para referirnos tanto a los países de América Latina como del Caribe. 
datos era la producción de autores latinoamericanos en revistas internacionales. Si bien esto es un inconveniente (por el reducido volumen), también es cierto que se trata de la porción de producción con más calidad, tal como muestran Moya-Anegón y Herrero-Solana (2002). Estos autores realizaron un análisis de cocitación de revistas en el que afloran revistas españolas y latinoamericanas citadas por los autores de la región cuando publican en revistas internacionales. Entre ellas se encuentran las españolas Revista Española de Documentación Científica y Boletín de $A N A B A D$ y las brasileñas Revista de Biblioteconomía de Brasilia y Ciência da Informação, las cuales son además caracterizadas temáticamente.

Siguiendo este principio de comportamiento, Herrero-Solana y Liberatore (2008) rastrearon las 34 revistas iberoamericanas de ByCI (18 españolas y 16 latinoamericanas) en los índices del ISI. A pesar de no ser usadas como fuentes, estas revistas pueden ser incluidas en las referencias de los trabajos. Esto permitió determinar la visibilidad de las mismas a partir del análisis de las citas recibidas en la bibliografía de los trabajos publicados en revistas recogidas por el ISI. Los resultados del estudio revelan que la Revista Española de Documentación Científica es lejos, con diferencia, la más citada; y que muy por debajo de ésta aparecen: Ciência da Informaçao, Boletin de $A N A B A D$, Revista de Biblioteconomía de Brasilia, Revista da Escola de Biblioteconomia da Universidade Federal de Minas Gerais, Revista de Bibliotecología y Documentación, Revista AIBDA, y Cybermetrics. Con menor visibilidad se encuentran las revistas Ciencias de la Información, Revista Brasileira de Biblioteconomia e Documentação, y el Boletin de la Asociación Andaluza de Bibliotecarios.

A pesar de que estos estudios brindan una idea aproximada sobre la revistas de la región, pocas son las bases de datos donde la cantidad de las mismas sea apreciable. Una alternativa es la base de datos LISA. Herrero-Solana y Quijano (2008) realizaron un estudio sobre el perfil temático de la revistas de la región en esta fuente. No obstante, LISA no recoge referencias bibliográficas, por lo que es imposible realizar estudios de citación a partir de ella.
También hay estudios centrados en una revista determinada. Moya-Anegón y Herrero-Solana (2001) analizaron la revista mexicana Investigación Bibliotecológica, encontrando que, dentro del contexto latinoamericano, es una revista de calidad, que oficia fundamentalmente como órgano de divulgación del Centro Universitario de Investigaciones Bibliotecológicas -CUIB-. Mueller y Pecegueiro (2001), y Liberatore, Herrero-Solana y Guimarães (2007) estudiaron la revista Ciência da Informação, destacando de ella su estabilidad en el tiempo y su capacidad para atraer artículos. Pinto et al (2006) analizaron las características de las citaciones realizadas por los autores que publican en dicha revista encontrando un alto porcentaje de autocitas, seguidas de citas a revistas internacionales indizadas por el ISI, y en tercer lugar revistas latinoamericanas incluidas en SCielo, Latindex, etc. entre las que se destacan Revista de Biblioteconomia de Brasilia, Transinformação, Revista da Escola de Biblioteconomia da Universidade Federal de Minas Gerais, su sucesora desde 1996, Perspectivas em Ciência da Informação, y Electronic Library.

Liberatore, et al (2003) realizaron un análisis de dominio de la revista Referencias, editada por la Asociación de Bibliotecarios Graduados de la República Argentina (ABGRA). Del análisis de las referencias bibliográficas incluidas en los trabajos publicados en la misma, los autores encontraron que cuatro de las diecisiete revistas más citadas proceden de países latinoamericanos. En orden decreciente de citas recibidas se encuentran: Ciência da Informação, Ciencias de la Información, Revista AIBDA y Revista de la Universidad de Antioquia.

En otros trabajos se estudiaron comparativamente grupos de revistas procedentes de la región latinoamericana e iberoamericana en términos de visibilidad e influencia. Mesa Fleitas, et al (2006) hicieron un estudio de la calidad y visibilidad de la revista cubana Ciencias de la Información, y la compararon con otras tres revistas de otros países de la región: Ciência da Informação, Investigación Bibliotecológica y Revista Interamericana de Bibliotecología. Encontraron que las dos primeras tenían más visibilidad en bases de datos de alcance internacional, 
que las últimas. Asimismo, mostraron que la revista Ciencias de la Información destaca tanto por su alta internacionalidad como por su importancia como vía de divulgación de los principales resultados de la actividad científico-informativa de los especialistas cubanos y de los de la región iberoamericana que publican en ella.

Gorbea Portal y Suárez Balseiro (2007) analizaron un grupo de 11 revistas procedentes de cinco países de la región, consideradas como las más productivas de la especialidad según la Base de datos Información y Bibliotecología Latinoamericana-INFOBILA-. El objetivo de dicho trabajo fue visualizar las relaciones de influencia, importancia y posición relativa de las revistas. Los autores concluyen que entre los países y las revistas existen relaciones de endogamia y escasa visibilidad, aunque destacan entre las revistas más citadas a: Actualidades de la Información Científica y Técnica (actual revista Ciencias de la Información), Revista da Escola de Biblioteconomia da Universidade Federal de Minas Gerais, Revista Brasileira de Biblioteconomía e Documentaçâo, Revista Interamericana de Bibliotecología, Investigación Bibliotecológica y Revista AIBDA.

En un reciente artículo Arencibia (2008) estudió la visibilidad en GS de la revista cubana ACIMED, comparándola con la de otras nueve revistas de lengua hispana. Cinco españolas, una mexicana, una peruana, una colombiana y otra cubana. Los resultados del estudio revelan que ACIMED se equipara en visibilidad a la Revista Española de Documentación Científica, El Profesional de la Información y Anales de Documentación.

Como hemos indicado anteriormente, no contamos con bases de datos de citas que contengan una nutrida oferta de revistas de ByCI de la región. SCOPUS no ha cambiado significativamente el escenario existente con las bases de datos del ISI. Sin embargo, la aparición de un motor de búsqueda gratuito y especializado en información científicoacadémica, Google Scholar, ha contribuido a que se convierta en una nueva fuente de datos para estudiar la visibilidad de la literatura.
Esta investigación se propone determinar la visibilidad en Google Scholar de un conjunto de revistas latinoamericanas de Bibliotecología y Ciencia de la Información, y clasificarlas según su grado de visibilidad en dicha fuente de datos.

\section{MATERIALES Y MÉTODOS}

\section{Identificación y selección de las revistas}

Las fuentes de datos utilizadas para la identificación y la selección de las revistas latinoamericanas de ByCI a incluir en este estudio fueron: el ULRICH'S Periodicals Directory; el Catálogo de LATINDEX y la selección de revistas más productivas según la base de datos INFOBILA realizada por Gorbea Portal y Suárez Balseiro (2007).

Del ULRICH se seleccionaron las revistas vigentes editadas en alguno de los países latinoamericanos, clasificadas como académico-científicas (Academic) Scholarly) dentro de la categoría temática "Library and Information Sciences". Por otra parte, del Catálogo de LATINDEX se eligieron las revistas vigentes editadas en algunos de los países de la región e incluidas en las categorías temáticas "Bibliotecología" o "Ciencias de la Información", respectivamente, y categorizadas como revistas de investigación, de divulgación científica o cultural, o técnico-profesionales. Se excluyeron revistas con contenidos efímeros como boletines de noticias, y las de temáticas englobadas en la categoría Ciencias de la Información pertenecientes a áreas afines como Periodismo y Comunicación, Informática, entre otras. Por último, se incluyeron las revistas seleccionadas por Gorbea Portal y Suárez Balseiro (2007) que estaban activas a la fecha del estudio. De ese grupo quedaron fuera dos revistas ya discontinuadas y una, denominada Bibliotecas, que no pudo identificarse cual era.

En total la muestra quedó conformada por 43 revistas. De cada una se guardaron los siguientes datos: Título, ISSN, Editor, País, Naturaleza, y año de inicio. Además se asignó una " $E$ " a las revistas con versión electrónica, las iniciales "OA" a las de acceso 
abierto y una " $R$ " a las que declaraban tener referato como proceso de admisión de las contribuciones.

\section{Método de análisis}

Cada una de las revistas seleccionadas se buscó en GS a través de la aplicación Publish or Perish (PoP) de Harzing's (http://www.harzing.com/pop.htm). GS es un buscador que permite recuperar artículos y otro tipo de documentos académico-científicos de una amplia variedad de editores académicos, sociedades profesionales, repositorios institucionales, etc.; en tanto que, PoP es un programa desarrollado especialmente para recuperar y analizar las citaciones académicas de los documentos recogidos en este buscador, calculando un conjunto de indicadores bibliométricos basados en el análisis de citación.

Las búsquedas se realizaron en la semana del 9 al 13 de febrero de 2009. Desde la opción Analysis Journal Impact se buscó por el nombre completo de la revista o por su abreviatura estandarizada. Cuando no había coincidencias se optó por la búsqueda por palabras en la casilla All of the words de la opción General Citation Search.

Para todas las revistas el recuento de citas se hizo considerando el período 1999-2008. En el caso de revistas con menos de diez años de existencia, la misma aplicación informática calculó el período de cobertura en función de la antiguedad de los documentos recuperados.

Cabe aclarar que una de las limitaciones que presenta la herramienta utilizada, y por ende la metodología empleada para recuperar las citas, radica en que la falta de normalización de los nombres de las revistas citadas puede dar lugar a omisiones no deseadas, como haber dejado fuera del estudio algunas citas, o incluso revistas, que por las razones expuestas y no pudieron ser detectadas. De igual modo, el peso de estas cuestiones en el resultado final puede considerarse despreciable.

Para las revistas citadas en GS se capturaron los siguientes indicadores bibliométricos calculados por el programa mencionado:
1. cantidad total de artículos recuperados;

2. cantidad total de citas recibidas;

3. promedio de citas por año (Cites/year);

4. promedio de citas por artículo (Cites/paper);

5. índice $\mathrm{h}$ (h-index)

6. índice h contemporáneo (hc-index)

7. índice h individual (hI-index),

8. índice h individual normalizado (hI norm)

9. AWCR, y

10. AW

Como ya se anticipara en la Introducción, el índice h es una medida propuesta por Hirsch (2005) para cuantificar la trayectoria de los investigadores, que matiza el número de documentos publicados con el número de citas recibidas. Se trata de una medida de impacto acumulado.

El índice h contemporáneo, desarrollado por Sidiropoulos, et al (2006), pondera cada artículo citado según la edad de los mismos, otorgando menos peso a los artículos más antiguos.

El índice h individual, desarrollado por Batista, et al (2006) relativiza el índice $h$ al número de autores firmantes, con el objeto de reducir los efectos de la coautoría sobre las citas recibidas. PoP también calcula una variante de éste, denominado índice $h$ individual normalizado, que normaliza el número de citaciones de cada artículo a partir del cociente entre el número de citas y el número de autores de cada artículo.

Por último, los índices AWCR y AW son variantes del índice AR propuesto por Jin (2007). AWCR mide el número de citas para un conjunto de artículos, ajustado a la edad de cada uno de los artículos individuales. Es una medida que representa el impacto relativo de un conjunto de artículos citados. AW es definido como la raíz cuadrada del anterior, y se utiliza con fines de comparación con el índice h.

Todas estas medidas, junto con la mención de las referencias a los trabajos en las que han sido propuestas, se encuentran explicadas en el manual 
del usuario del programa PoP (Publish or Perish User's Manual, 2009).

Con los indicadores 3 a 10 se confeccionó una matriz de datos, y se calculó la correlación utilizando el coeficiente $\mathrm{R}^{2}$. Luego, se aplicaron dos técnicas de análisis multivariado de datos: análisis de cluster y escalamiento multidimensional (MDS). La primera de ellas permite transformar un gran número de variables correlacionadas en un número reducido de ellas, no correlacionadas, llamadas componentes principales, con el objetivo de mejorar la interpretabilidad de los datos. La segunda permite clasificar y representar en forma de árbol jerárquico un cúmulo de información y organizarla en clusters o clases en base a criterios de similaridades o distancias, y la tercera, es una técnica que permite encontrar los objetos más significativos sobre la base de criterios de proximidad y minimización de las distancias, para luego representarlos como puntos en un espacio de dimensiones reducidas. Con los resultados obtenidos se elaboraron dos mapas bidimensionales en el que se muestran las revistas agrupadas en función de su visibilidad relativa dentro del conjunto estudiado (alta, media y baja). Por último se analizó la relación entre el grado de visibilidad y el país de procedencia de las revistas, el sistema de admisión de las publicaciones (con o sin referato) y el soporte (electrónico o impreso).

\section{RESULTADOS}

De las 43 revistas seleccionadas (anexo 1), 31 tienen visibilidad en GS, entendiendo por visibles a las revistas cuyos artículos recibieron al menos una cita dentro del período considerado (1999-2008). Con los valores de las 31 revistas se construyó una matriz (anexo 2) a la que se le aplicaron diferentes técnicas multidimensionales. En primer lugar se aplicó escalamiento muldimensional (MDS) en cuyo mapa (figura 1) se identifican claramente 4 grupos distintos de revistas.

El grupo 1 (G1), ubicado en el cuadrante superior izquierdo del mapa, está integrado por 15 revistas que presentan los mejores valores en todos los indicadores. En orden decreciente del valor del índice AW se encuentran: ACIMED; Perspectivas em Ciência da Informação, DataGramaZero; Informação \& Sociedade: Estudos; Transinformação; Encontros Bibli: Revista de Biblioteconomia e Ciencia da Informação; Ciência da Informação; Investigación Bibliotecológica: Archivonomía, Bibliotecología e Información; C L E I Electronic Journal; Revista ACB; Revista Interamericana de Bibliotecología; Información, Culturay Sociedad; ETD - Educação Temática Digita; Ciencias de la Información y Biblioteca universitaria.

El grupo 2 (G2), en el cuadrante inferior izquierdo del mapa, se compone de 7 revistas con valores intermedios. Estas son: Revista Brasileira de Biblioteconomia e Documentação; Revista Digital de Biblioteconomía e Ciência da Informação; Pez de Plata; Arquivistica.net; Biblios; InfodiversidadyEnl@ce.

Los grupos 3 (G3) y 4 (G4), ubicados en los cuadrantes inferior y superior derechos, respectivamente, están integrados por las revistas con los valores más bajos. En el grupo 3 se encuentran: Informação \& Informação; Revista AIBDA, Liber: Revista de Bibliotecología y F@ro. En el grupo 4 aparecen la Revista Interamericana de Nuevas Tecnologías de la Información; Simbiosis: revista electrónica de ciencias de la información; Revista Argentina de Bibliotecología; Acceso. Revista Puertorriqueña de Bibliotecología y Documentación, y Revista da Escola da Biblioteconomía da Universidade Federal de Minas Gerais.

La diferencia entre estos dos últimos grupos radica principalmente en su potencial, ya que mientras de visibilidad. Mientras que las revistas que integran el grupo 3 tienen mayor cantidad de artículos potencialmente citables, las del grupo 4 tienen una pobre presencia, ya que en su mayoría no pasan de un solo artículo. El caso de la Revista da Escola da Biblioteconomía da Universidade Federal de Minas Gerais merece una mención especial porque en 1996 fue sustituída por Perspectivas em Ciência da Informação, que se encuentra en el G1, por lo que su escasa visibilidad estaría vinculada a este hecho. De igual modo los autores decidieron incluirla en los análisis por la reiterada presencia del título en los estudios previos encontrados en la literatura. 
Sandra Miguel / Víctor Herrero_Solana

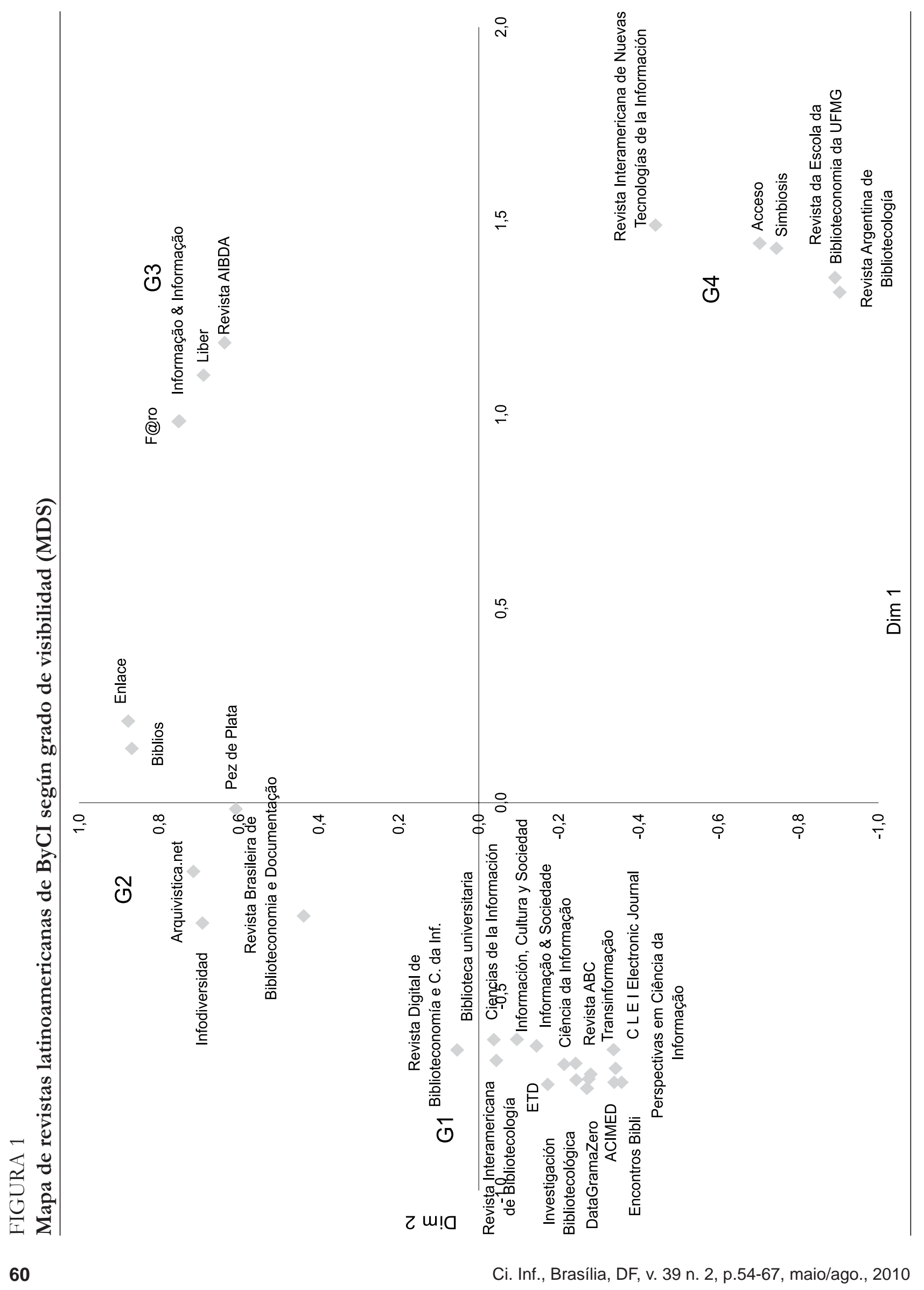


Dendograma de revistas latinoamericanas de BCI según grado de visibilidad - Análisis de cluster *

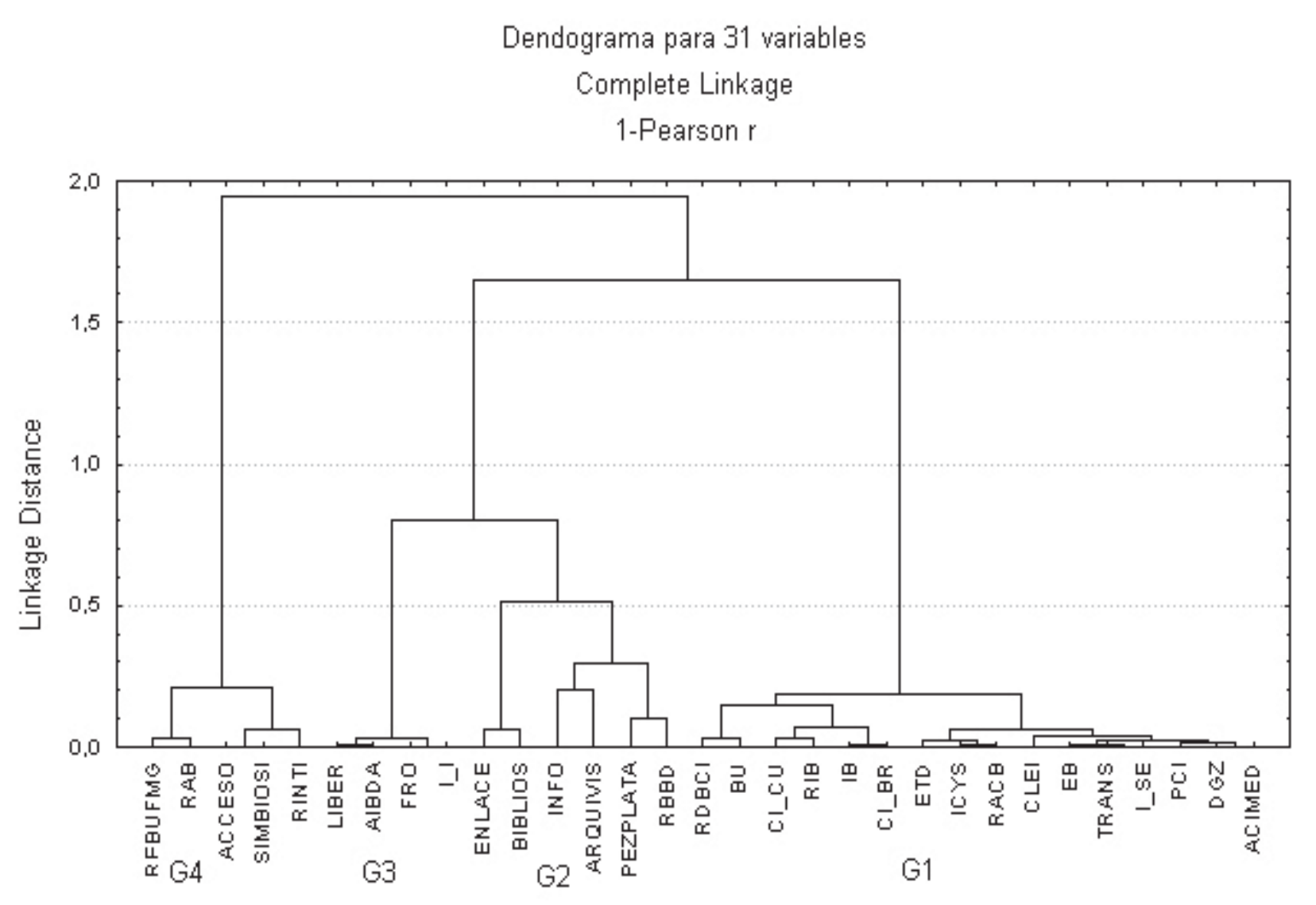

* Los títulos de las revistas están codificados por razones de espacio. Ver nombres desarrollados en Anexo 2.

Para complementar los resultados del MDS utilizamos el análisis de cluster, pudiendo observar que los grupos son más distantes cuanto más se diferencian en su grado de citación. Ello es, las revistas del grupo 1 (alta visibilidad) son las que más se distancian de las del grupo 4 (baja visibilidad) -figura 2-. A distancias intermedias entre uno y otro grupo aparecen las revistas de los grupos 2 y 3.

En un intento por determinar si el país de procedencia de las revistas (Tabla 1), la modalidad de admisión de los artículos, con o sin referato (Tabla 2), o el soporte, electrónico o impreso (Tabla 3), incide sobre la citación de las revistas, se calcularon las frecuencias de éstas en función de esas tres categorías de análisis y el grado de visibilidad (alta, media y baja).

Ci. Inf., Brasília, DF, v. 39 n. 2, p.54-67, maio/ago., 2010
TABLA 1

Visibilidad de las revistas según país de origen

\begin{tabular}{ccccc}
\hline \multicolumn{5}{c}{ Nro. de revistas según grado de visibilidad } \\
\hline & alta & media & baja & Total \\
\hline Brasil & 8 & 3 & 2 & 13 \\
México & 2 & 0 & 1 & 3 \\
Argentina & 1 & 1 & 1 & 3 \\
Costa Rica & 0 & 0 & 1 & 1 \\
Puerto Rico & 0 & 0 & 2 & 2 \\
Chile & 1 & 1 & 1 & 3 \\
Cuba & 2 & 0 & 0 & 2 \\
Perú & 0 & 1 & 0 & 1 \\
Colombia & 1 & 0 & 1 & 2 \\
Venezuela & 0 & 1 & 0 & 1 \\
Total & 15 & 7 & 9 & 31 \\
\hline
\end{tabular}


TABLA 2

Visibilidad de las revistas según modalidad de admisión de los artículos

\begin{tabular}{ccccc}
\hline \multicolumn{5}{c}{ Nro. de revistas según grado de visibilidad } \\
\hline & alta & media & baja & Total \\
\hline Con referato & 12 & 6 & 3 & 21 \\
Sin referato & 3 & 1 & 6 & 10 \\
Total & 15 & 7 & 9 & 31 \\
\hline
\end{tabular}

TABLA 3

Visibilidad de las revistas según soporte

\begin{tabular}{ccccc}
\hline \multicolumn{5}{c}{ Nro. de revistas según grado de visibilidad } \\
\hline & alta & media & baja & Total \\
\hline Electrónicas & 15 & 7 & 5 & 27 \\
Impresas & 0 & 0 & 4 & 4 \\
Total & 15 & 7 & 9 & 31 \\
\hline
\end{tabular}

Conforme a los resultados obtenidos, no parece haber correlación alguna entre el grado de visibilidad y el país de origen de las revistas. Parecería haber una cierta correlación entre la visibilidad y el sistema de admisión de los trabajos (con o sin referato), ya que las primeras son más citadas que las segundas. Finalmente, y tal como se esperaba, se observan diferencias notables entre el grado de citación y la disponibilidad o no de versión electrónica. Aquellas revistas existentes en Internet tienen una capacidad de captar citas mucho mayor que aquellas que no lo están.

Todas las revistas del grupo 1 (de alta visibilidad) disponen de versión electrónica, y excepto el caso de Ciencias de la Información, el resto ofrece acceso gratuito al texto completo de los artículos. Se trata además de revistas con una antiguedad de diez o más años. En el grupo 2, de visibilidad media, también todas las revistas tienen versión electrónica. Exceptuando el caso de la Revista Brasileira de Biblioteconomia e Documentação el resto de las revistas son relativamente nuevas, con una antiguedad de diez años o menos. Por el contrario, de las revistas con más baja visibilidad (grupos 3 y 4) hay tres (Revista Interamericana de Nuevas Tecnologias de la Información, Revista Argentina de Bibliotecología y Liber) que solo cuentan con versión impresa, y su antiguedad es diversa.

\section{CONCLUSIONES Y DISCUSIÓN}

Los resultados de este estudio permiten identificar la visibilidad en GS de un conjunto de revistas latinoamericanas del campo de la ByCI, seleccionadas sobre la base de criterios de vigencia, calidad y productividad.

El principal aporte de estos resultados radica en brindar a los investigadores del campo de la ByCI, y en especial, a los de la región latinoamericana, la posibilidad de reconocer a-priori las revistas en las que sería más conveniente difundir los resultados de sus investigaciones, teniendo en cuenta las posibilidades de visibilidad de los mismos. Conforme a los hallazgos de este estudio los trabajos publicados en las revistas del grupo 1 tendrían más probabilidad de ser conocidos, leídos y citados que los publicados en las revistas de los otros tres grupos, al menos desde la perspectiva de su visibilidad en GS.

Este estudio también puede ser de interés para los editores de las revistas, en aras de mejorar la calidad de las mismas, y/o de implementar medidas que contribuyan a un incremento de su influencia en la comunidad científica regional e internacional. En este sentido, la disponibilidad de revistas en soporte electrónico es vista como una oportunidad para incrementar esta influencia (Arencibia, 2008), y aunque los resultados de este estudio parecen estar en concordancia con esta tendencia, para medir su significación habría que ampliar el tamaño de la muestra.

En comparación con los resultados de estudios previos encontramos algunas coincidencias que ponen de relieve, sin duda alguna, la mayor influencia que tienen ciertas revistas de la región por sobre otras. Tales son los casos de la revista brasileña Ciência da Informação, la cubana Ciencias de la Información, y en menor medida la mexicana Investigación Bibliotecológica y la colombiana Revista Interamericana de Bibliotecología. Son revistas que se destacan en diversos análisis encontrados en la literatura, y coincidentemente también aparecen en el grupo de las más visibles en 
nuestro estudio. No obstante, también encontramos algunas discrepancias. Así por ejemplo, mientras la Revista $A I B D A$ aparece entre las publicaciones latinoamericanas más citadas en varios trabajos anteriores (Liberatore, 2003; Gorbea Portal, 2007; Herrero-Solana y Liberatore, 2008), en nuestro estudio la misma aparece en el grupo de revistas de escasa visibilidad. Una situación similar se da con la Revista da Escola da Biblioteconomia da Universidade Federal de Minas Gerais, que en trabajos previos aparece como una de las más citadas dentro del conjunto de revistas de la región (Gorbea Portal y Suárez Balseiro, 2007; Herrero-Solana y Liberatore, 2008), y en nuestro estudio tiene muy bajo impacto. Sin embargo, en este caso se debe a que dicho título fue reemplazado por Perspectivas em Ciência da Informação, que sí aparece en el grupo de las más visibles. Asimismo, nuestro trabajo revela la existencia de varias revistas con alta visibilidad relativa en GS que ni se mencionan en trabajos anteriores, o que aparecen, en su defecto, con escasa presencia. Algunos ejemplos son los casos de Perspectivas em Ciência da Informação; Datagramazero; Encontros Bibli; Informação \& Sociedade: estudos; Información Cultura y Sociedad; Revista A C B: biblioteconomía em Santa Catarina; CLEI, entre otras.

Una mención particular merece la revista $A C I M E D$. Los resultados de este estudio la muestran como la más visible del conjunto estudiado; en un trabajo previo basado en la misma fuente de datos, Arencibia (2008) la calificó como una revista de una influencia equiparable a varias publicaciones españolas de alcance internacional. Sin embargo, resulta llamativo el hecho de que en ninguno de los trabajos anteriores, basados en análisis de citas, esta revista no aparezca siquiera mencionada. También hay que tener en cuenta que es una revista que se encuentra a caballo entre la ByCI y las ciencias de la salud, siendo estas últimas disciplinas en el que las tasas de citación son incomparablemente más altas que en las primeras.

Estas discrepancias podrían evidenciar que la evaluación de la visibilidad de las revistas latinoamericanas de ByCI está influida en parte por la fuente de datos utilizada para realizar los estudios, y por la falta de uniformidad en los criterios de selección de las mismas, habida cuenta de las dificultades para su identificación y valoración de su calidad, ya señaladas.

Respecto de la fuente de datos utilizada en este estudio, GS, si bien no es un sustituto equivalente a las bases de datos bibliográficas reconocidas internacionalmente como SCOPUS o las del Web of Science del ISI, ha empezado a cobrar un amplio reconocimiento como medio de difusión de la literatura académico-científica, y por tanto, se está convirtiendo en una poderosa herramienta para la evaluación de la visibilidad de los resultados de investigación. Muestra de ello lo constituye el desarrollo y amplia utilización de PoP, una aplicación específica para el análisis de citas basado en esta fuente de datos. Si bien es cierto que este programa tiene como principal desventaja la falta de mecanismos de normalización de las citas, y que por lo tanto puede introducir sesgos no deseados a la hora de la localización de las revistas o de los recuentos, también lo es que la falta de normalización es un problema común a la mayoría de las fuentes de datos y un tema recurrente en la mayoría de los estudios basados en el análisis de citas. La aplicación tiene, sin embargo, importantes ventajas como la posibilidad de calcular de una manera fácil y rápida un conjunto de indicadores que basados en el recuento de citas contribuyen a determinar y comparar la visibilidad relativa de un conjunto de revistas, sobre la base de medidas que relacionan la frecuencia de citación con el volumen de la producción, la antiguedad y los efectos de la coautoría.

Artigo submetido em 08/05/2009 e aceito em 10/12/2010. 
Sandra Miguel / Víctor Herrero_Solana

\section{REFERENCIAS}

ARENCIBIA JORGE, Ricardo. Acimed en Scholar Google: un análisis de citas de la Revista Cubana de los Profesionales de la Información y la Comunicación en la Salud. Acimed, La Habana, v. 18, n. 1, 2008. Disponível em: <http://scielo.sld.cu/pdf/aci/v18n1/ aci03708.pdf> Acesso em: 5 feb. 2009.

BATISTA, Pablo D.; CAMPITELI, Monica G.; KINOUCHI, Osame; MARTINEZ, Alexandre S. Is it possible to compare researchers with different scientific interests? Scientometrics, Budapest, v. 68, n. 1, p. 179-189, 2006.

BIHUI, Jin. The AR-index: complementing the h-index. ISSI Newsletter, v. 3, n.1, p. 6, 2007.

GARCÍA DE LEÓN, Alicia Ma. Lista de publicaciones en Bibliotecología y Ciencias de la Información en curso en América Latina. Ciencias de la Información, La Habana, v. 32, n. 2, p. 59-67, agosto, 2001.

GÓMEZ FUENTES, H. Las revistas latinoamericanas de Bibliotecología y Ciencias de la Información bajo el prisma de los servicios bibliográficos internacionales. Investigación Bibliotecológica, México, v. 14, n.7, p. 27-32, 1993.

GORBEA-PORTAL, Salvador; SUÁREZ-BALSEIRO, Carlos A. Análisis de la influencia y el impacto entre revistas periféricas no incluidas en el Science Citation Index. Revista Interamericana de Bibliotecología, Medellin, v. 30, n. 2, p. 47-70, 2007.

Disponível em: <Disponible en: http:/ /www.scielo.org.co/pdf/rib/ v30n2/v30n2a03.pdf> Acesso em: 3 feb. 2009.

HERRERO-SOLANA, Víctor; RÍOS-GÓMEZ, Claudia. Producción latinoamericana en biblioteconomía y documentación en el Social Science Citation Index (SSCI) 1966-2003. Information Research: an internacional electronic journal, Sheffield. v. 11, n. 2 paper 247, 2006.

HERRERO-SOLANA, Víctor; LIBERATORE. Gustavo. Visibilidad internacional de las revistas iberoamericanas de Bibliotecología y Documentación. Revista Española de Documentación Científica, v. 31, n. 2, p. 230-239, 2008.

HERRERO-SOLANA, Víctor; QUIJANO-SOLÍS, Álvaro. Perfil temático de la investigación iberoamericana en bibliotecología y documentación a través de LISA. In ENCUENTRO ASOCIACIÓN DE EDUCADORES E INVESTIGADORES DE BIBLIOTECOLOGÍA, ARCHIVOLOGÍA, CIÊNCIA DE LA INFORMACÍON Y DOCUMENTACIÓN DE IBEROAMÉRICA Y EL CARIBE, 8., México. Anais... México 8, 2008, México: CUIBUNAM, 12-14 nov. 2008.

HIRSCH, J. E. An index to quantify an individual's scientific research output. 2005. Disponível em: < http://arxiv.org/abs/physics/0508025> Acesso em: 9 feb. 2009.

LIBERATORE, Gustavo; CORINGRATO, Marcela; AMERIO, Adrián. Estudio de la producción profesional en Bibliotecología en la Argentina: análisis de dominio de la revista Referencias. Transinformação, Campinas, v. 15, n. 2, p. 221-229, maio/ago. 2003.

LIBERATORE, Gustavo; HERRERO-SOLANA, Víctor; GUIMARÃES, J. A. C. Bibliometric analysis of the Brazilian journal 'Ciência da Informação' during the period of 2000-2004. Brazilian Journal of Information Science, Marília, São Paulo, v. 1, n. 2, p. 3-21, jul./dec. 2007.
MALTRÁS BARBA, B. Los indicadores bibliométricos: fundamentos y aplicación al análisis de la ciencia. Gijón, Asturias: Ediciones TREA, 2003.

MARTIN, B. R.; IRVING, J. Asessing basic research: Some partial indicators of scientific progress in radio astronomy. Research Policy. v. 12 , n. 2, p. $61-90,1983$.

MESA FLEITAS, María Elena; RODRÍGUEZ SÁNCHEZ, Yaniris; SOLÓRZANO ÁLVAREZ, Ernestina; TARANGO ORTIZ, Javier. Revista cubana Ciencias de la Información: indicadores de edición y presentación, productividad y visibilidad en los últimos 14 años de su edición. In: CONGRESSO INTERNACIONAL DE INFORMACIÓN, 2006. Anais... Intempres, 2006. Disponível em: <http://www.congreso-info.cu/UserFiles/File/Info/ Intempres2006/Intempres2006/Ponencias/97.pdf>. Acesso em 12 feb. 2009.

MOYA-ANEGÓN, Félix de; HERRERO-SOLANA, Víctor. Análisis de dominio de la revista mexicana Investigación bibliotecológica. Información, Cultura y Sociedad, Buenos Aires, n. 5, p. 10-28, 2001.

- Visibilidad internacional de la producción científica iberoamericana en Biblioteconomía y Documentación (1991-2000). Ciência da Informação, Brasilia, v. 31, n. 3, p. 54-65, set./dez. 2002. Disponível em: http://revista.ibict.br/ciinf/index.php/ciinf/ article/view/150/129 Acesso em: 12 feb. 2009.

MUELLER, S. P. M.; PECEGUEIRO, C. M. P. D. A. O periódico Ciência da Informação na década de 90: um retrato da área refletido em seus artigos. Ciência da Informação, Brasilia, v. 30, n. 2, p. 47-63, maio/ago. 2001. Disponível em: < http://revista.ibict.br/ciinf/index. php/ciinf/article/view/193/170> Acesso em: 15 feb. 2009.

PINTO, Adilson Luiz; RODRÍGUEZ BARQUÍN, Beatriz-Ainhize; MOREIRO GONZÁLEZ, José Antonio. Análisis de citación de la revista Ciência da Informação del Ibict. Ciência da Informação, Brasilia, v. 35, n. 3, p. 153-165, set./dez. 2006. Disponível em: < http://www. scielo.br/pdf/ci/v35n3/v35n3a15.pdf> Acesso em: 20 feb. 2009.

PUBLISH OR PERISH USER'S MANUAL. 1990-2009. Melbourne: Tarma Software Research.

RAMIREZ LEYVA, E. 1994. La producción latinoamericana como apoyo al desarrollo de la región. In: IFLA GENERAL COUNCIL, 60., 1994. Proceedings... Milan: IFLA, 2009. Disponível em: <http:// www.ifla.org/IV/ifla60/60-rame.htm>. Acesso: 13 feb. 2009.

SCImago. 2007. SJR — SCImago Journal \& Country Rank. Disponível em: <http://www.scimagojr.com> Acesso em 13 feb. 2009.

SIDIROPOULOS, Antonis; KATSAROS, Dimitiros; YANNIS, Manolopoulos.. Generalized h-index for disclosing latent facts in citation networks. Scientometrics, v. 72, n.2, p. 253-280, 2007.

URBIZAGASTEGUI ALVARADO, Ruben. Las revistas de bibliotecología y ciencias de la información en América Latina. Biblios: Revista electrónica de bibliotecología, archivología y museología, Lima, v. 7, n. 24, abr./jun. 2006. Disponível em: <http://redalyc.uaemex. $\mathrm{mx} /$ redalyc/pdf/161/16172407.pdf> Acesso em: 8 ene. 2009 . 
Visibilidad de las revistas latinoamericanas de bibliotecología y ciencia de la información a través de Google Scholar

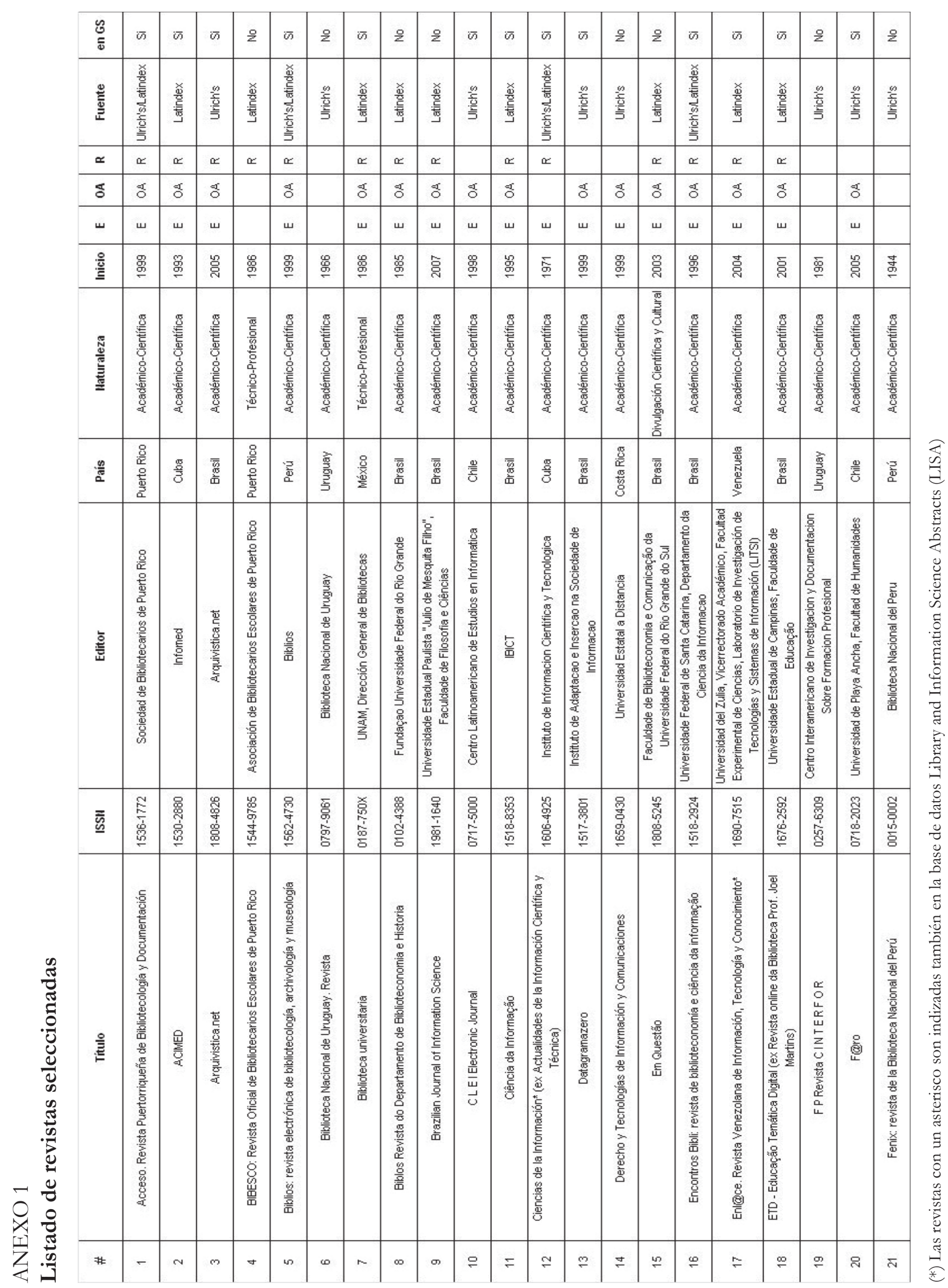


Sandra Miguel / Víctor Herrero_Solana

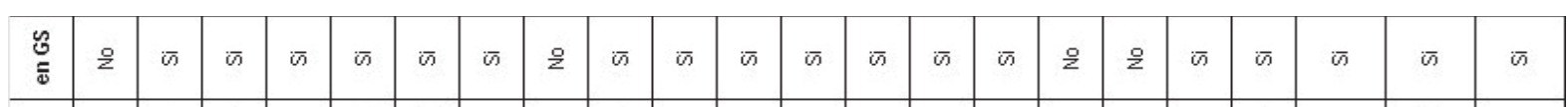

\begin{tabular}{|c|c|c|c|c|c|c|c|c|c|c|c|c|c|c|c|c|c|c|c|c|c|c|}
\hline 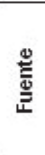 & 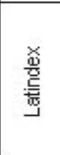 & 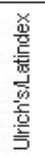 & 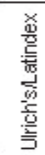 & $\begin{array}{l}\frac{m}{\underline{\underline{c}}} \\
\frac{\underline{\underline{m}}}{2}\end{array}$ & 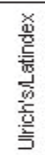 & 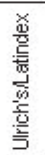 & $\begin{array}{l}\frac{\text { 黑 }}{\underline{\underline{\underline{m}}}}\end{array}$ & 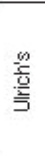 & 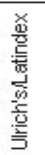 & $\begin{array}{l}\frac{\underline{\underline{c}}}{\underline{\underline{\underline{w}}}}\end{array}$ & 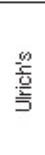 & 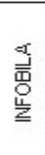 & 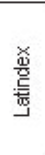 & 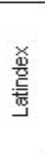 & 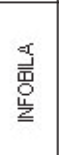 & 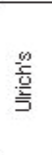 & 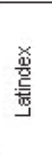 & 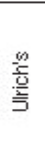 & 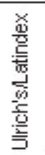 & 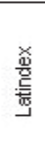 & 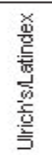 & $\frac{\frac{g}{2}}{\frac{\underline{\underline{y}}}{3}}$ \\
\hline$\propto$ & & $\propto$ & $\propto$ & $\alpha$ & $\propto$ & $\propto$ & & $\propto$ & $\propto$ & & $\propto$ & & & $\propto$ & & & & $\propto$ & a & & $\propto$ & $\propto$ \\
\hline$\delta$ & & ฮ์ & ฮ์ & ฮ็ & ฮ์ & ฮ็ & & & б์ & f & ฮิ & ઠీ & & ฮ์ & & & & ฮี & ฮ์ & & 항 & ฮf \\
\hline س & & ш & ш & ш & u & ш & & & ш & ш & ш & ш & & ш & & & & ш & ш & & ш & ш \\
\hline 退 & 兽 & 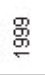 & $\stackrel{\stackrel{9}{g}}{\circ}$ & $\stackrel{\text { g }}{\text { s. }}$ & $\stackrel{\text { g }}{\stackrel{g}{\circ}}$ & $\begin{array}{l}\stackrel{9}{\circ} \\
\stackrel{\circ}{\circ}\end{array}$ & 兽 & 总 & $\underset{\text { ్ㅗ }}{2}$ & 兽 & 兽 & 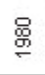 & $\underset{\stackrel{g}{g}}{\stackrel{g}{g}}$ & 管 & 喜 & $\begin{array}{l}\stackrel{9}{\%} \\
\end{array}$ & $\begin{array}{l}\stackrel{g}{g} \\
\sigma\end{array}$ & 兽 & 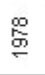 & 畸 & 莒 & $\stackrel{g}{\stackrel{g}{\circ}}$ \\
\hline
\end{tabular}

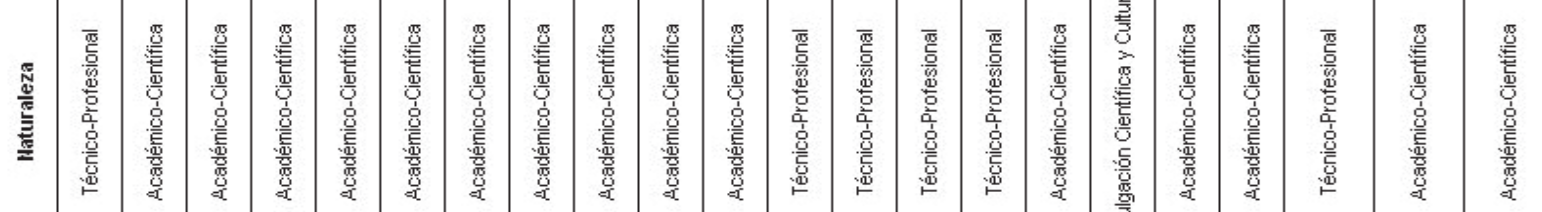

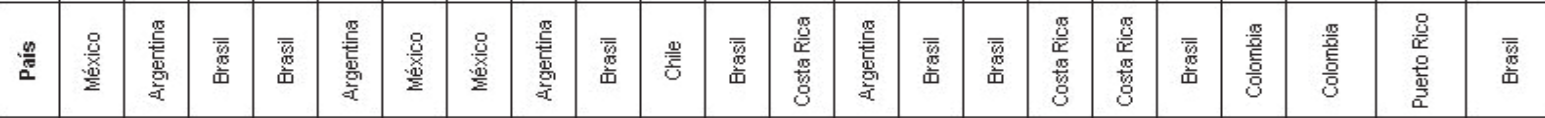

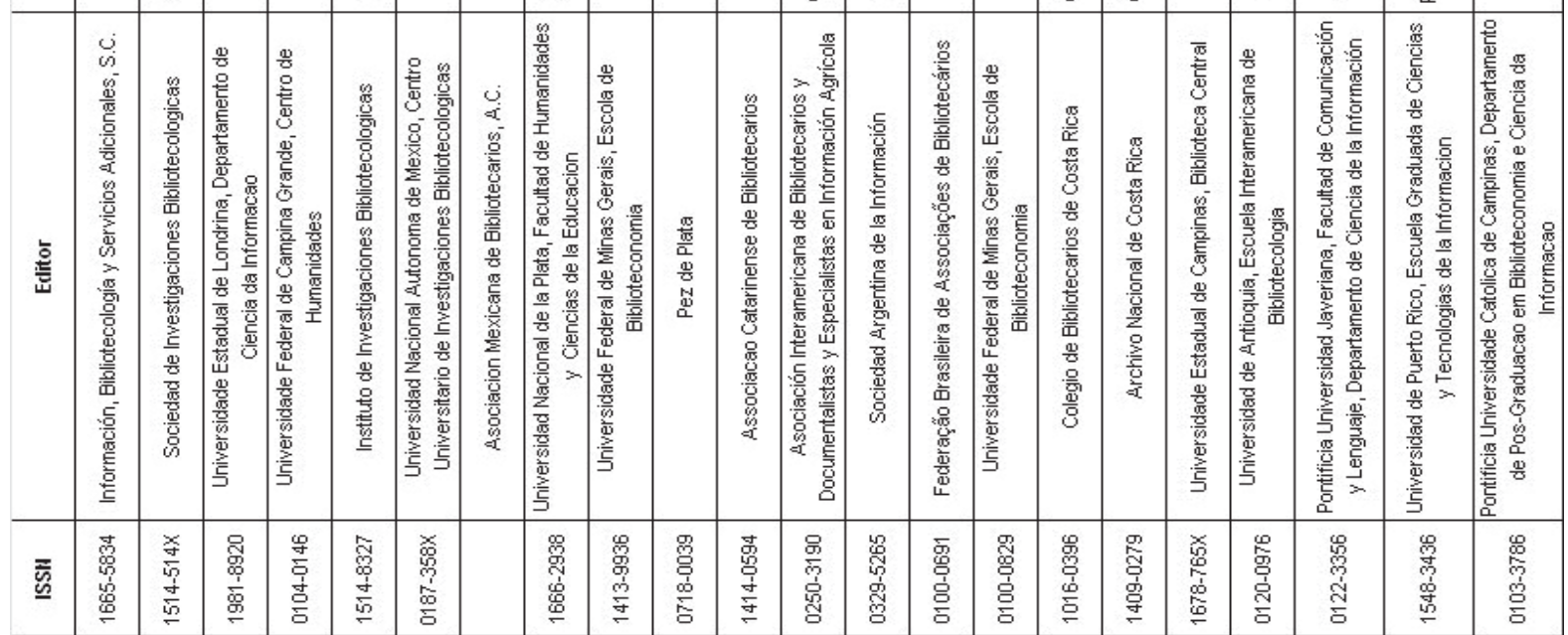

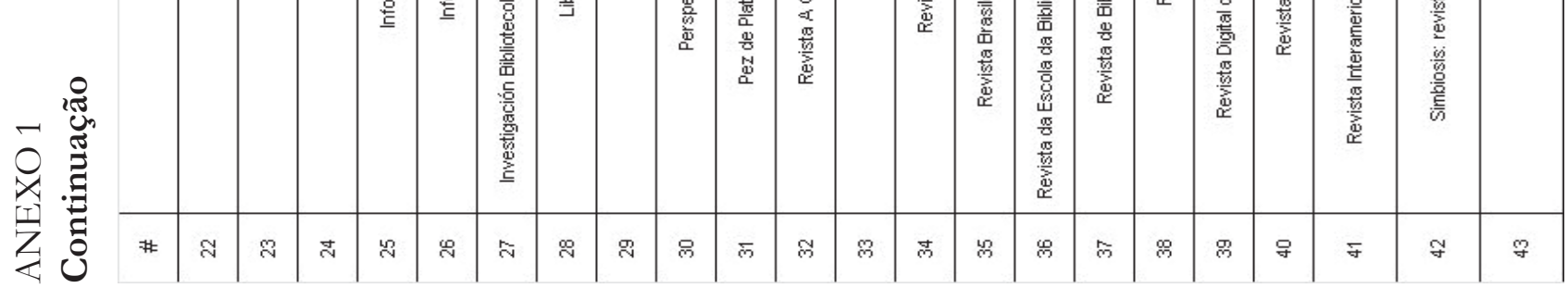


Visibilidad de las revistas latinoamericanas de bibliotecología y ciencia de la información a través de Google Scholar

\begin{tabular}{|c|c|c|c|c|c|c|c|c|c|c|c|c|c|c|c|c|c|c|c|c|c|c|c|c|c|c|}
\hline 5 & - & 总 & 兽 & 导 & $\frac{\pi}{\infty}$ & 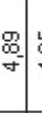 & zo & t & $=\frac{\mathrm{g}}{\mathrm{g}}$ & 点 & $\begin{array}{l}R \\
\stackrel{2}{\sim}\end{array}$ & & & & 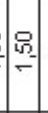 & 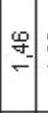 & 을 & 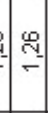 & $\stackrel{m}{=}$ & $\stackrel{8}{\simeq}$ & 垔 & : & $\begin{array}{l}5 \\
0 \\
0\end{array}$ & 5 & & \\
\hline & $\overline{5}$ & $\begin{array}{l}\text { तิ } \\
\text { 号 }\end{array}$ & 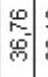 & & & & & 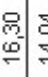 & 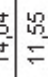 & न & $E$ & & & & ה্য & $\frac{N}{\sim}$ & & $\stackrel{9}{\circ}$ & $\stackrel{-}{=}$ & בי & $\frac{9}{3}$ & בים & & 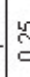 & & \\
\hline $\bar{\Xi}$ & 焉 & 查 & 量 & 焉 & 总 & $\begin{array}{l}\text { 量 } \\
5\end{array}$ & 量 & $\begin{array}{l}\text { So } \\
\text { वे }\end{array}$ & 票 & 总 & 孚 & 受 & 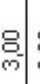 & & ì & 兽 & 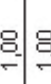 & - & S: & 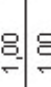 & O & $\stackrel{\text { O }}{-}$ & 을 목 & 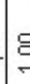 & & \\
\hline 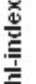 & 量 & $\frac{7}{i 5}$ & $\begin{array}{l}g \\
g \\
\oplus\end{array}$ & $\begin{array}{l}\stackrel{2}{8} \\
\mathrm{v}\end{array}$ & $\begin{array}{l}\text { 号 } \\
\text { - }\end{array}$ & $\begin{array}{l}\text { go } \\
\mathrm{f}\end{array}$ & & & $=$ & 照- & 㝵 & & & & $=$ & 空 & 善 & 害 & $\stackrel{\mathrm{g}}{-}$ & 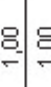 & $\stackrel{8}{-}$ & $\stackrel{\mathrm{g}}{-}$ & 농믐 & 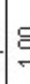 & & $=$ \\
\hline 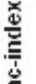 & 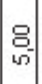 & 馝 & 查 & 喜 & 害 & 查 & 兽 & 品 & 㝵 & 总 & 㝵 & $\stackrel{8}{-}$ & & & is & 总 & 总突 & 害 & 㝵 & 영믐 & 要 & $\stackrel{\mathrm{g}}{-}$ & 은 & 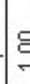 & & \\
\hline 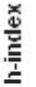 & 品 & 查 & 量 & 查 & 曼 & 焉 & 总 & 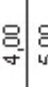 & 量 & 㝵 & 号 & & & 部 & is & 㝵 & 总 & 㝵 & $\stackrel{g}{-}$ & 음믐 & 要 & 焉 & 은 & 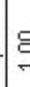 & 骂 & \\
\hline
\end{tabular}

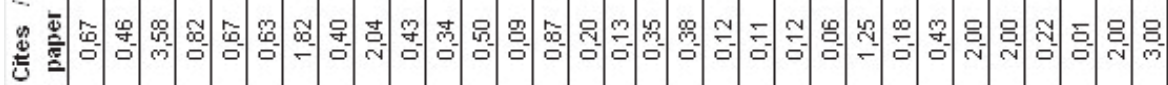

\begin{tabular}{|c|c|c|c|c|c|c|c|c|c|c|c|c|c|c|c|c|c|c|c|c|c|c|c|c|c|}
\hline 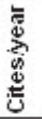 & 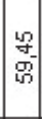 & 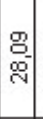 & & $\begin{array}{l}\text { जิ } \\
\text { क- }\end{array}$ & $\begin{array}{l}9 \\
\stackrel{9}{\circ} \\
\stackrel{\infty}{\circ}\end{array}$ & $\begin{array}{c}\stackrel{g}{9} \\
\stackrel{m}{\sim}\end{array}$ & $\stackrel{ }{\mathrm{z}}$ & $\begin{array}{l}\stackrel{S}{\infty}- \\
=-\end{array}$ & \begin{tabular}{c|c}
0 \\
0 \\
$\infty$ \\
$\infty$
\end{tabular} & & $\begin{array}{ll}5 \\
0 \\
0\end{array}$ & 9 & 点 & 츨 & $\stackrel{\overline{-}}{-}$ & $\begin{array}{l}F= \\
=-5\end{array}$ & $=\frac{19}{9}$ & $\stackrel{\text { Oㅇ }}{-}$ & $\begin{array}{lll}0 \\
0 \\
-5\end{array}$ & $\stackrel{-}{a}$ & $\stackrel{\mathrm{N}}{-}$ & - & & & \\
\hline 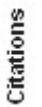 & $\begin{array}{l}\text { 量 } \\
\text { 总 } \\
\text { | }\end{array}$ & 查 & $\begin{array}{l}\text { 拿 } \\
\stackrel{0}{2}\end{array}$ & $\begin{array}{l}\text { 연 } \\
\text { in }\end{array}$ & $\begin{array}{l}\text { 害 } \\
\text { 品 }\end{array}$ & 要 & $\begin{array}{l}\text { 歖 } \\
\text { - }\end{array}$ & $\begin{array}{l}\text { 总 } \\
\text { 号 }\end{array}$ & 离 & $\begin{array}{l}\text { 量 } \\
\text { s. }\end{array}$ & 5 & $\frac{5}{5}$ & 음 & 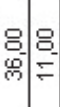 & $\frac{\mathrm{O}}{\mathrm{N}}$ & 品 & 鼌 & $\stackrel{\text { S }}{=}$ & $\begin{array}{c}\text { 查量 } \\
\end{array}$ & 总 & 总总 & 空 & 웜 & $\stackrel{8}{=}$ & \\
\hline
\end{tabular}

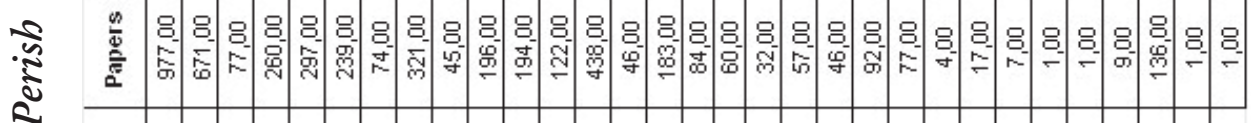

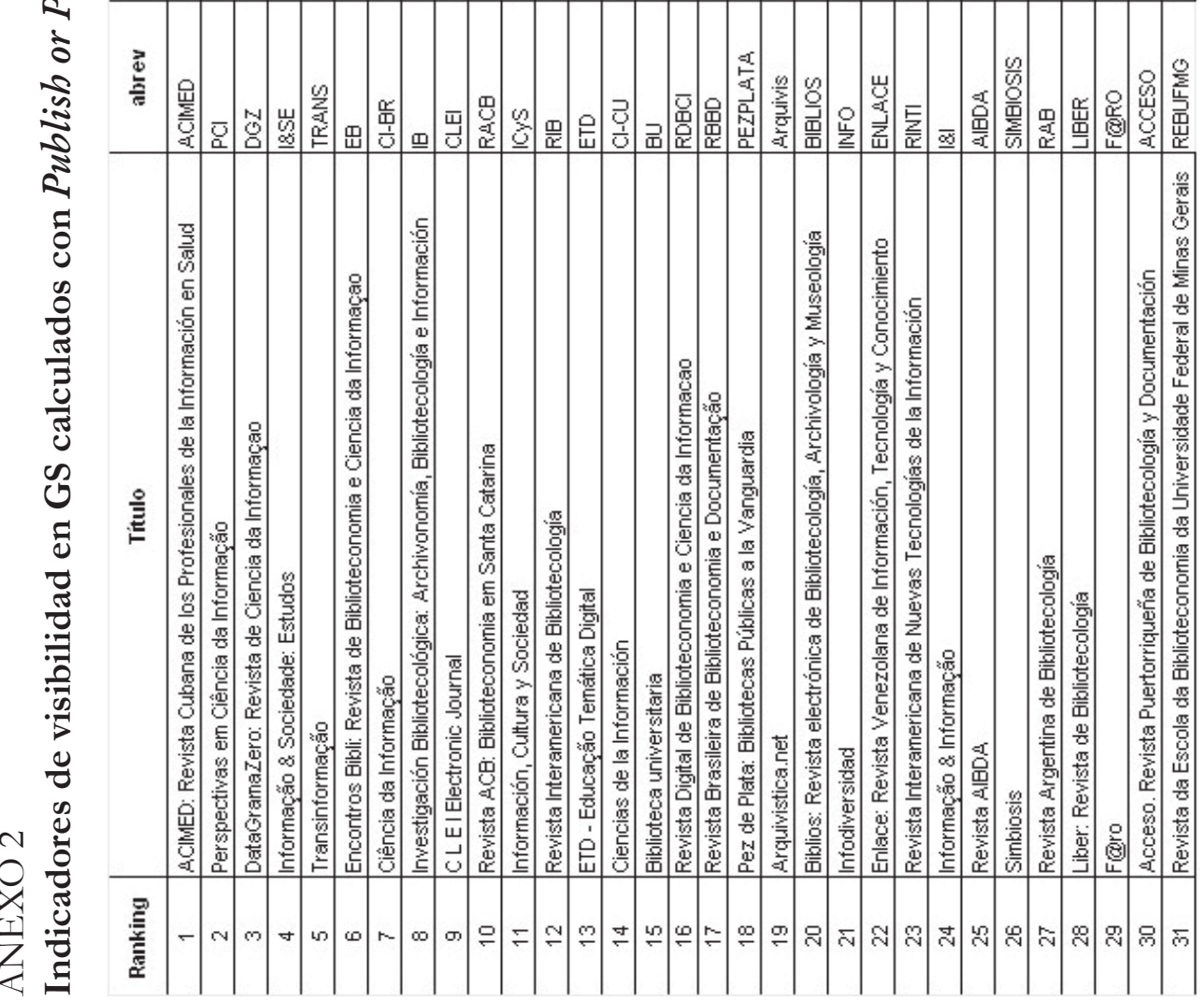

Ci. Inf., Brasília, DF, v. 39 n. 2, p.54-67, maio/ago., 2010 